\title{
Are Treatment Preferences Relevant in Response to Serotonergic Antidepressants and Cognitive- Behavioral Therapy in Depressed Primary Care Patients? Results from a Randomized Controlled Trial Including a Patients' Choice Arm
}

\author{
Roland Mergl ${ }^{\mathrm{a}}$ Verena Henkel ${ }^{\mathrm{b}}$ Antje-Kathrin Allgaier ${ }^{\mathrm{c}}$ Dietmar Kramer ${ }^{\mathrm{a}}$ \\ Martin Hautzinger ${ }^{d}$ Ralf Kohnen ${ }^{e}$ James Coyne ${ }^{f} \quad$ Ulrich Hegerla \\ ${ }^{a}$ Department of Psychiatry, University of Leipzig, Leipzig, Departments of ${ }^{b}$ Psychiatry and ${ }^{\circ}$ Child and Adolescent \\ Psychiatry, Ludwig Maximilian University of Munich, Munich, dDepartment of Clinical Psychology, Eberhard

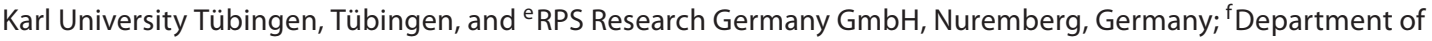 \\ Psychiatry, University of Pennsylvania Health System, Philadelphia, Pa., USA
}

\section{Key Words}

Treatment preference $\cdot$ Sertraline $\cdot$ Cognitive-behavioral therapy $\cdot$ Primary care $\cdot$ Depression

\begin{abstract}
Background: Little is known about the influence of depressed patients' preferences and expectations about treatments upon treatment outcome. We investigated whether better clinical outcome in depressed primary care patients is associated with receiving their preferred treatment. Methods: Within a randomized placebo-controlled single-centre 10-week trial with 5 arms (sertraline; placebo; cognitive-behavioral group therapy, CBT-G; moderated self-help group control; treatment with sertraline or CBT-G according to patients' choice), outcomes for 145 primary care patients with mild-to-moderate depressive disorders according to DSM-IV criteria were investigated. Preference for medication versus psychotherapy was assessed at screening using a single item. Post-baseline difference scores for the Hamilton De-
\end{abstract}

\section{KARGER}

Fax +41613061234 E-Mail karger@karger.ch www.karger.com
(C) 2010 S. Karger AG, Base

0033-3190/11/0801-0039\$38.00/0

Accessible online at:

www.karger.com/pps pression Rating Scale (HAMD-17) were used to assess treatment outcome (mixed-model repeated-measures regression analysis). Results: Depressed patients receiving their preferred treatment $(n=63)$, whether sertraline or CBT-G, responded significantly better than those who did not receive their preferred therapy $(n=54 ; p=0.001)$. The difference in outcome between both groups was 8.0 points on the HAMD-17 for psychotherapy and 2.9 points on the HAMD-17 for treatment with antidepressants. Results were not explained by differences in depression severity or dropout rates. Conclusions: Patients' relative preference for medication versus psychotherapy should be considered when offering a treatment because receiving the preferred treatment conveys an additional and clinically relevant benefit (HAMD17: +2.9 points for drugs; +8.0 points for $(B \mathrm{BT}-\mathrm{G})$ in outcome.

Copyright $\odot 2010$ S. Karger AG, Basel

Clinical trials registration number: NCT00226642.
Dr. Roland Mergl

Department of Psychiatry, University of Leipzig

Semmelweisstr. 10, DE-04103 Leipzig (Germany)

Tel. +49 34197 24556, Fax +493419724539

E-Mail Roland.Mergl@medizin.uni-leipzig.de 


\section{Introduction}

In routine care, therapy of depression is complicated by the fact that providers, especially in primary care, have to deal with patients' disease concepts and treatment preferences which can considerably differ from providers' own conceptions and preferred treatments [1-3]. So, it is relevant to study how important the fit is between patients' treatment preference and the treatment offered for the outcome. Also the option to choose a particular therapy instead of being assigned to it by randomization might have an effect on the outcome. The findings of the few available studies are controversial.

Thornett $[4]$ reviewed studies $[5,6]$ investigating the effects of patient preference in trials of treatment of depressive disorders in a general practice setting and concluded that patients who received treatment according to their preference did not significantly differ from those who were randomized. These results were confirmed by a comprehensive meta-analysis of 32 medical and psychological treatment studies [7].

Leykin et al. [8] examined treatment outcomes of patients who got their preferred treatment via randomization, and those who did not. To examine this question, they used clinical data from a double-blind randomized placebo-controlled trial comparing antidepressant medication to cognitive therapy for outpatients suffering from moderate-to-severe depressive disorders [9]. Most of the 174 investigated patients with available data stated a preference for one treatment over the other. Nevertheless, there were no significant differences in treatment outcome between patients who got their treatment of choice versus those who did not. Therefore, the authors concluded that treatment preference was not a key issue in determining treatment outcome.

Kocsis et al. [10] found patient preference to be a relevant moderator of treatment response for patients suffering from chronic forms of major depressive disorders: patients who preferred drugs had higher remission rates and lower depression scores at the last study visit when receiving medication than when receiving psychotherapy and vice versa. Their analysis based on data from $429 \mathrm{pa}-$ tients who were included in a randomized multicenter trial of nefazodone, the Cognitive Behavioral Analysis System of Psychotherapy (CBASP) or combination therapy, and who gave information about their treatment preference at study entry. However, the validity of this study is limited by a low percentage of patients with a clear preference for the monotherapies and the absence of a placebo group.
At baseline assessment in a study of enhanced care for depression that emphasized improving patient adherence to medication [11], patients were classified as either receptive or non-receptive to antidepressants based on their response to a 4-point Likert scale. Almost half of the patients $(47.4 \%)$ were not receptive to antidepressants and the intervention did not prove cost-effective for them. In contrast, for patients indicating receptivity to medication, there was a substantial incremental cost-effectiveness ratio. In a recent study, Raue et al. [12] addressed the question of whether treatment preferences are associated with clinical outcome in primary care patients suffering from major depressive disorder. Sixty patients were randomly assigned to treatment congruent or incongruent with their treatment preference stated at study entry. Treatments were 20 weeks of escitalopram or 12 weekly sessions of interpersonal psychotherapy. Patients expressed stronger preference for interpersonal psychotherapy than for escitalopram and strength of treatment preference was found to be more closely associated with the clinical outcome than the congruence versus incongruence of treatment preference with the assigned therapy. These results are encouraging, but clearly preliminary due to the limited sample size.

According to the study protocol, we analyzed the data from the MinD study [13] - a randomized placebocontrolled single-centre 10 -week trial with 5 treatment arms - concerning the following pre-specified question:

Does treatment outcome differ according to whether patients receive their preferred treatment (sertraline or cognitive-behavioral group therapy, CBT-G)?

In addition, the following secondary research questions were addressed:

(1) How are treatment preferences in terms of pharmacological or psychological treatment in primary care patients suffering from mild-to-moderate depressive disorders?

(2) Do primary care patients with a drug preference differ from those with a preference for psychological treatment concerning demographic and clinical variables?

(3) Are differences in outcome explained by the way of getting the preferred treatment: by randomization or by choice?

(4) Does treatment outcome in the placebo group differ in patients who prefer drugs and patients who prefer psychotherapy?

(5) Are there any pronounced differences between matched patients (receiving their preferred treatment) and mismatched patients (who do not receive their preferred treatment) in the number of dropouts and the mean number of attended visits? 


\section{Materials and Methods}

\section{Study Design}

The aim of the study was to investigate the efficacy of an antidepressant (sertraline) and CBT-G for treatment of primary care patients with mild-to-moderate depressive disorders. The adult eligible patients were randomly assigned to $5 \mathrm{arms}, 3$ of which are relevant for this analysis: (1) sertraline (initial daily dose: $50 \mathrm{mg}$ with an escalation in dose in steps of $50 \mathrm{mg}$ up to $200 \mathrm{mg}$ per day being possible at weeks 2, 4 and 6); (2) short-term CBT-G with 9 weekly sessions at $90 \mathrm{~min}$ each with 5-8 members after an initial individual 50-min session [for details see, 14]; (3) the patient randomized in this arm could choose (patients' choice arm; PC) either a treatment with sertraline or with CBT-G. Since this article deals with effects of treatment preferences on outcome, primarily data from the subjects in the active treatment arms were considered. Results for the patients randomized to placebo were analyzed in secondary analyses.

Sertraline and placebo capsules were identical in appearance so that blinding was ensured. The duration of the guided self-help group (GSG) was as long as the treatment in the other arms (10 weeks) after an initial individual session with an average duration of 50-min nine weekly group sessions (of $1.5 \mathrm{~h}$ each) were conducted. In the GSG, participants had the opportunity to talk about their personal situation and their daily life in a supportive atmosphere. However, the group leader was not free to conduct any psychotherapeutic intervention in the GSG sessions. All CBT$\mathrm{G}$ and GSG were videotaped.

Blinding concerning CBT-G versus the psychotherapy control condition was not possible. Therefore, the analysis of the effects of treatment preferences on outcome in the psychotherapy control condition does not make sense, more so as it cannot be determined whether all patients can be expected not to recognize it as a control condition rather than as a preferred treatment. For this reason, we did not consider the guided self-help groups in our analyses.

To be enrolled in the study, the patients had to meet the following criteria: a minimum age of 18 years; a diagnosis of subthreshold (minor) depression, dysthymia or major depressive disorder or depressive disorder not otherwise specified with mild to moderate severity and Hamilton Rating Scale for Depression (HAMD-17) [15] total scores $\geq 8$ and $\leq 22$. There are also several exclusion criteria, explained by Hegerl et al. [13]. A rather representative sample of depressed primary care patients could be included, based on referrals by primary care providers and screening of primary care patients, inclusion of patients with depressive disorders not otherwise specified, with minor depression and with comorbid anxiety and somatoform disorders as well as the entry criterion of a HAMD-17 total score $>7$, but $<23$. Eight points in the HAMD-17 was selected as minimum baseline total score in our inclusion criteria because lower scores indicate absence of depression [16].

Data were collected between May 2000 and November 2004. Our study protocol complied with the revised Declaration of Helsinki [17] and Good Clinical Practice guidelines. An independent Ethics Review Committee approved the study and all subjects gave written informed consent after the procedure and possible side effects were fully explained.

\section{Clinical Diagnostics}

Patients were diagnosed by trained psychiatrists and clinical psychologists according to DSM-IV criteria [16]. The diagnoses based on the German computerized version [DIA-X; 18] of the fully structured Composite International Diagnostic Interview [19]. This interview was supplemented by a section for the assessment of minor depression according to DSM-IV research criteria.

\section{Preference Measures}

Before randomization, participants were asked to complete an inventory to measure attitudes toward psychotherapy with the original title: 'Fragebogen zur Messung der Psychotherapiemotivation' [FMP; 20]. The FMP consists of 47 items and 4 subscales.

To obtain a measure for the preference of psychotherapy versus drugs, item 36 was chosen to represent the General Treatment Preference (FMP-TP): 'I prefer an efficient drug or a successful operation to psychological treatment.'

Five possible answers are given and only 1 answer has to be marked with a cross by the patient: 1 = fully endorsed; 2 = endorsement somewhat restricted; $3=$ undetermined; $4=$ tendency not to agree; $5=$ not true at all.

Subjects with FMP-TP item 36 scores $\leq 2$ were identified as patients with a drug preference, subjects with FMP-TP item 36 scores $\geq 4$ as patients with a psychotherapy preference, whereas patients scoring 3 on this item were considered as having 'no clear treatment preference'.

Based on the FMP-TP item-score and the received treatment, the patients were divided in 2 groups. Matched to treatment of choice 'yes' (matched: receiving sertraline or CBT-G in accordance with the preference) and matched to treatment of choice 'no' (mismatched: receiving sertraline or CBT-G not in accordance with the preference) - independently of whether they were randomly assigned to a certain treatment (sertraline, CBT-G) or had the chance to choose their treatment (PC: sertraline or CBT-G).

\section{Outcome Measures}

For the present study, the HAMD-17 [15] was chosen as the primary efficacy measure, the standard clinician-administered interview for the assessment of depression severity. All HAMD-17 evaluations were carried out by 2 blinded psychologists. Three rater training programs were performed and a 95\% rater agreement was achieved.

Secondary outcomes were response and remission rates. Responders were defined as patients with a HAMD-17 reduction at the individual's end of treatment of $\leq 50 \%$ and HAMD-17 endpoints $<8$, remitters as patients with HAMD-17 endpoints $<8$ [13].

\section{Data Analyses}

Explorative statistical analysis was performed with data from the intent-to-treat population comprising all randomized patients.

\section{Analysis of Patient Characteristics}

We conducted $\chi^{2}$ tests to test differences in demographic (gender) and clinical characteristics (diagnosis, psychiatric comorbidity, drug and psychotherapy pretreatment in the last 2 years, number of dropouts) between preference groups for statistical significance. Analogously, analyses of variance were applied for metrical variables (age, number of attended visits) and Kruskal-Wallis tests for ordinal variables (HAMD-17 baseline total scores). 
Table 1. Number of patients with or without preferences for either drug treatment or psychotherapy, grouped by the therapy received

\begin{tabular}{llrl}
\hline & \multicolumn{2}{l}{ Treatment preference } \\
\cline { 2 - 4 } & $\begin{array}{l}\text { medication } \\
(\mathrm{n}=32)\end{array}$ & $\begin{array}{c}\text { indecisive } \\
(\mathrm{n}=28)\end{array}$ & $\begin{array}{l}\text { psychotherapy } \\
(\mathrm{n}=85)\end{array}$ \\
\hline Sertraline $(\mathrm{n}=51)$ & $11(21.6)$ & $10(19.6)$ & $30(58.8)$ \\
CBT-G $(\mathrm{n}=39)$ & $5(12.8)$ & $7(17.9)$ & $27(69.2)$ \\
Patient preference arm: decision for CBT $(\mathrm{n}=20)$ & $5(25.0)$ & $1(5.0)$ & $14(70.0)$ \\
Patient preference arm: decision for sertraline $(\mathrm{n}=35)$ & $11(31.4)$ & $10(28.6)$ & $14(40.0)$ \\
\hline
\end{tabular}

Figures in parentheses are percentages.

Using a two-sided $\chi^{2}$ test, we addressed the question of whether matched and mismatched patients significantly differed in the number of dropouts according to the study protocol.

\section{Analysis of Outcome Differences}

We applied a mixed-model repeated-measures (MMRM) regression analysis [21], using all available data from visits (baseline, week 2, 4, 6, 8 and 10) to analyze outcome differences. This approach allowed us to use all available data, is very flexible in time effect modeling and can appropriately handle missing data [22]. Our mixed linear model used the restricted maximum likelihood algorithm and the HAMD-17 total scores as the dependent variable. It included random effects for intercept and slope as well as fixed class effects for treatment (CBT-G, sertraline), treatment preference (FMP-TP $\geq 4$ psychotherapy preference, FMP-TP $\leq 2$ drug preference), visit week and the interaction of these factors. Regarding the factor 'treatment', the patients who could choose their treatment (PC arm) and those who were randomized in the corresponding arms (sertraline, CBT-G) were summarized; thus, patients with compatible and incompatible treatment expectations could be found in the enlarged CBT-G and sertraline groups.

We used statistical significance of the interaction of treatment (sertraline, CBT-G), treatment preference (FMP-TP $\geq 4$ psychotherapy preference, FMP-TP $\leq 2$ drug preference) and visit week to indicate whether the rates of change during treatment were different in matched versus mismatched patient groups.

In an analogous way, differences between matched and mismatched patients in therapy outcome were assessed within the placebo condition.

The power of our study to detect a medium (Cohen's $d=0.50)$ effect size given the sample sizes (mismatch: $<\mathrm{n}=54$; match: $<\mathrm{n}=63$ ) and assuming a one-tailed t test for independent sample comparison with an $\alpha$ of 0.05 computed with $\mathrm{G}^{*}$ Power $^{\mathrm{TM}}$, version 3.0.5 [23] was 0.85 .

A value of $p \leq 0.05$ was considered to be statistically significant. Statistical analysis was performed by using SPSS software, version 12.0 [24] as well as STATA version 8.0 for mixed model analysis [25].

\section{Results}

\section{Patients' Characteristics and Treatment Preferences}

From 1,099 primary care patients screened by primary care providers in one German region (Nuremberg), 368 patients fulfilled the inclusion criteria of the MinD study. In total, 145 patients remained for the present analyses: 51 of 83 patients from the sertraline arm, 39 of 61 patients from the CBT-G arm and 55 of 82 patients from the PC arm. The distribution of the answer categories for FMPTP item 36 in these groups is shown in table 1.

Of the 145 patients, $32(22.1 \%)$ had a stronger preference for drugs, and 85 a stronger preference for psychotherapy (58.6\%). For sample characteristics, see table 2.

Comparison of the 3 categories 'medication' $(n=32)$, 'psychotherapy' ( $\mathrm{n}=85)$ and 'indecisive regarding treatment preference' $(n=28)$ revealed significant baseline differences in the distribution of main psychiatric diagnoses. Patients with depressive disorders not otherwise specified more often had no clear treatment preferences (FMP item $36=3$; 42.9\%) than patients with major depressive disorder (32.1\%) or double depression (25\%). Matched and mismatched patients did not significantly differ in demographic and clinical variables at baseline (table 2).

\section{Outcome Differences}

The MMRM regression analysis $(\mathrm{n}=117)$ resulted in a significant preference $\times$ treatment $\times$ time interaction $(\beta=-0.89 ; 95 \% \mathrm{CI}=-1.39$ to $-0.38 ; \mathrm{z}=-1.39 ; \mathrm{p}=0.001)$. Figure 1 reveals that this effect is due to patients in the sertraline group having better therapy outcomes if they had a stronger preference for drugs than for psychotherapy, whereas patients in the CBT-G group had a more fa- 
Table 2. Patient characteristics

\begin{tabular}{|c|c|c|c|c|c|c|c|}
\hline & \multicolumn{4}{|c|}{ Treatment preference } & \multicolumn{3}{|c|}{ Match to the treatment of choice } \\
\hline & $\begin{array}{l}\text { medication } \\
(\mathrm{n}=32)\end{array}$ & $\begin{array}{l}\text { psychotherapy } \\
(\mathrm{n}=85)\end{array}$ & $\begin{array}{l}\text { indecisive } \\
(\mathrm{n}=28)\end{array}$ & $\mathrm{p}$ & $\begin{array}{l}\text { yes } \\
(n=63)\end{array}$ & $\begin{array}{l}\text { no } \\
(\mathrm{n}=54)\end{array}$ & $\mathrm{p}$ \\
\hline Age, years (mean $\pm \mathrm{SD})$ & $50.0 \pm 14.8$ & $43.4 \pm 13.1$ & $44.5 \pm 15.5$ & $0.08^{1}$ & $47.4 \pm 14.3$ & $42.7 \pm 12.8$ & $0.07^{4}$ \\
\hline Females & $21(65.6)$ & $56(65.9)$ & $17(60.7)$ & $0.88^{2}$ & $45(71.4)$ & $32(59.3)$ & $0.17^{2}$ \\
\hline HAMD-17 baseline total score, median (IQR) & $16(13-20)$ & $16(12-20)$ & $17(14.3-20.8)$ & $0.41^{3}$ & $16(13-20)$ & $16(13-20)$ & $0.84^{5}$ \\
\hline Diagnoses & - & - & - & $0.03^{*, 2}$ & - & - & $0.21^{2}$ \\
\hline Major depressive disorder & 7 (21.9) & $28(32.9)$ & $9(32.1)$ & - & $18(28.6)$ & $17(31.5)$ & - \\
\hline Dysthymic disorder & $1(3.1)$ & $4(4.7)$ & $0(0)$ & - & $1(1.6)$ & $4(7.4)$ & - \\
\hline Double depression $^{\mathrm{a}}$ & $19(59.4)$ & $34(40)$ & $7(25)$ & - & $28(44.4)$ & $25(46.3)$ & - \\
\hline Depressive disorder not otherwise specified & $3(9.4)$ & $16(18.8)$ & $12(42.9)$ & - & $14(22.2)$ & $5(9.3)$ & - \\
\hline Minor depressive disorders ${ }^{\mathrm{b}}$ & $2(6.3)$ & $3(3.5)$ & $0(0)$ & - & $2(3.2)$ & $3(5.6)$ & - \\
\hline Psychiatric comorbidity ${ }^{c}$ & $8(25)$ & $21(24.7)$ & $5(17.9)$ & $0.74^{2}$ & $16(25.4)$ & $13(24.1)$ & $0.87^{2}$ \\
\hline Drug pretreatment in the last 2 years & $8(25)$ & $26(30.6)$ & $8(28.6)$ & $0.84^{2}$ & $20(31.7)$ & $14(25.9)$ & $0.49^{2}$ \\
\hline Pretreatment with psychotropic drugs in the last 2 years & $8(25)$ & $22(25.9)$ & $8(28.6)$ & $0.95^{2}$ & $16(25.4)$ & $14(25.9)$ & $0.95^{2}$ \\
\hline Psychotherapeutic pretreatment in the last 2 years & $1(3.1)$ & $8(9.4)$ & $4(14.3)$ & $0.31^{2}$ & $5(7.9)$ & $4(7.4)$ & $1^{6}$ \\
\hline Number of dropouts & $12(37.5)$ & $33(38.8)$ & $7(25)$ & $0.41^{2}$ & $23(36.5)$ & $22(40.7)$ & $0.64^{2}$ \\
\hline Number of attended visits (mean \pm SD) & $4.9 \pm 1.6$ & $5.0 \pm 1.5$ & $5.5 \pm 0.9$ & $0.51^{3}$ & $4.9 \pm 1.6$ & $5.2 \pm 1.4$ & $0.24^{5}$ \\
\hline
\end{tabular}

Figures in parentheses are percentages unless otherwise specified. ${ }^{*} \mathrm{p} \leq 0.05$.

${ }^{1}$ Univariate ANOVA $;{ }^{2} \chi^{2}$ test $;{ }^{3}$ Kruskal-Wallis test; ${ }^{4} \mathrm{t}$ test for independent samples; ${ }^{5}$ Mann-Whitney test; ${ }^{6}$ Fisher's exact test.

${ }^{a}$ Dysthymic disorder with a current major depressive episode.

${ }^{\mathrm{b}}$ A group of subsyndromal depressive disorders (minor depression according to DSM-IV research criteria; mixed anxiety and depressive disorder; mixed anxiety and depressive reaction; persistent mood disorders not otherwise specified).

${ }^{c}$ According to Composite International Diagnostic Interview [19], diagnoses of anxiety and/or somatoform disorders.

vorable therapy response curve if their preference for psychotherapy was greater than their preference for drugs. A MMRM subgroup analysis indicates for patients treated with sertraline a significant treatment preference $\times$ time interaction $(\beta=0.62 ; 95 \% \mathrm{CI}=0.08-1.15 ; \mathrm{z}=2.25 ; \mathrm{p}=$ $0.025)$ as well as for patients receiving $\mathrm{CBT}-\mathrm{G}(\beta=-1.19$; $95 \% \mathrm{CI}=-2.05$ to $-0.33 ; \mathrm{z}=-2.72 ; \mathrm{p}=0.007$ ), with the latter effect being even more pronounced.

Considering the HAMD-17 total scores at the end of treatment, a moderate effect size of $d=0.42(95 \% \mathrm{CI}=$ 0.004-0.83) in favor of matched patients was found.

The difference in outcome (HAMD-17; week 10) between receiving or not receiving the preferred treatment as revealed by MMRM was 8.04 points for psychotherapy $(\mathrm{n}=51 ; 95 \% \mathrm{CI}=4.00-12.07$ points; $\mathrm{p}<0.001)$ and 2.86 points for pharmacotherapy ( $\mathrm{n}=66 ; 95 \% \mathrm{CI}=-0.22$ to 5.93 points; $\mathrm{p}=0.07)$. In line with these results, response and remission rates in matched patients treated with CBT-G (39.0\% and 43.9\%, respectively) were significantly higher than in mismatched patients receiving CBT-G $\left(0 \%\right.$; response rates: $\chi^{2}=5.69$; d.f. $=1 ; \mathrm{p}=0.017$; remission rates: $\chi^{2}=6.79$; d.f. $=1 ; \mathrm{p}=0.009$ ), whereas response and remission rates in matched versus mismatched patients treated with sertraline were comparable. Response and remission rates in matched as well as mismatched patients treated with sertraline or CBT-G are summarized in table 3.

Within the placebo group, the differences between matched and mismatched patients in the intensity of therapeutic changes failed to be statistically significant, as reflected by the corresponding match (yes/no) $\times$ time interaction $(\beta=0.47 ; 95 \% \mathrm{CI}=-0.14$ to $1.09 ; \mathrm{z}=1.51 ; \mathrm{p}=$ $0.13)$.

\section{Effects of Randomization and Choice}

Being randomized in a patient preference arm or not had no marked influence on outcome difference between patients who received their preferred treatment and those who did not, as indicated by a match (yes/no) $\times$ treatment choice $(y e s / n o) \times$ time interaction not being significant $(\beta=0.19 ; 95 \% \mathrm{CI}=-0.61$ to $0.99 ; \mathrm{z}=0.47 ; \mathrm{p}=$ $0.64)$.

MMRM analysis of the 63 patients receiving their preferred treatment revealed that having the possibility to choose the preferred treatment instead of being assigned to it by randomization had no significant additional effect on treatment outcome (PC $\times$ time interaction: $\beta=$ $0.14 ; 95 \% \mathrm{CI}=-0.77$ to $1.04 ; \mathrm{z}=0.30 ; \mathrm{p}=0.77)$. 

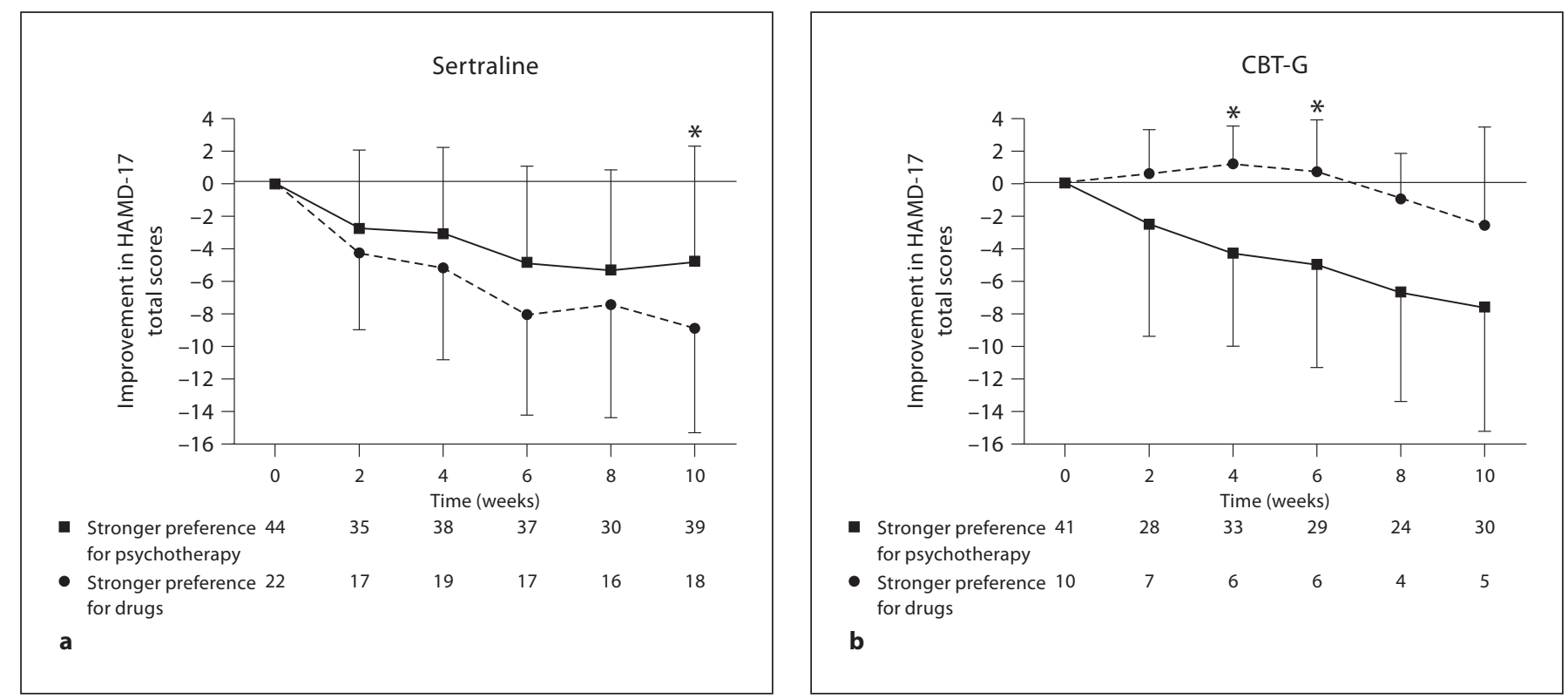

Fig. 1. Improvement differences in mean HAMD-17 total scores (intent-to-treat population; observed case analysis) between patients with preference for drugs versus psychotherapy for sertraline (a) and CBT-G (b). ${ }^{*} \mathrm{p} \leq 0.05$, two-sided independent-sample t test.

Table 3. Response and remission rates of depressed primary care patients who had received their preferred treatment (match) or not (mismatch), grouped by the therapy received

\begin{tabular}{|c|c|c|c|c|c|c|}
\hline & \multicolumn{3}{|c|}{ Response rate } & \multicolumn{3}{|c|}{ Remission rate } \\
\hline & match & mismatch & $\mathrm{p}$ & match & mismatch & $\mathrm{p}$ \\
\hline Sertraline $^{1}(n=66)$ & $8 / 22(36.4)$ & $18 / 44(40.9)$ & 0.72 & $10 / 22(45.5)$ & $19 / 44(43.2)$ & 0.86 \\
\hline $\mathrm{CBT}-\mathrm{G}^{2}(\mathrm{n}=51)$ & $16 / 41(39.0)$ & $0 / 10(0)$ & $0.02^{*}(\mathrm{FET})$ & $18 / 41(43.9)$ & $0 / 10(0)$ & $0.009^{* *}(\mathrm{FET})$ \\
\hline
\end{tabular}

Figures in parentheses are percentages. FET $=$ Fisher's exact test (which was chosen if expected values were $<5$; otherwise, $\chi^{2}$ tests for 2 independent samples were applied). ${ }^{*} \mathrm{p} \leq 0.05 ;{ }^{*} \mathrm{p} \leq 0.01$.

${ }^{1}$ Patients who could choose their treatment and had chosen sertraline as well as those who were randomized in the sertraline arm were summarized.

${ }^{2}$ Patients who could choose their treatment and had chosen CBT-G as well as those who were randomized in the CBT-G arm were summarized.

\section{Analysis of Dropouts}

In addition, it was examined whether the differences between matched and mismatched patients in the number of dropouts and the mean number of attended visits were significant. This was not the case (number of dropouts: two-sided $\chi^{2}$ test: $\chi^{2}=0.22$; d.f. $=1 ; \mathrm{p}=0.64$; mean number of attended visits: two-sided Mann-Whitney test, $\mathrm{Z}=-1.17 ; \mathrm{p}=0.24$ ).

\section{Discussion}

The main finding of this study is that treatment preference is clinically relevant for treatment outcome among primary care patients. The effect size computed for the differences between matched and mismatched patients in final depression scores is moderate (Cohen' $\mathrm{s} d=0.42$ ) and resembles that found in other studies [8]. This is 
noteworthy as very dissimilar treatments have been compared in our study. Similar effects were found in patients with chronic forms of major depressive disorder [10].

For patients treated with CBT-G, we found a significant and clinically relevant additional benefit of being matched to the treatment of choice (8 points in HAMD17). In line with this, the response rates were also higher in those receiving CBT-G (39.0 vs. $0 \%$ ).

These findings are in line with other results indicating that in general open-mindedness for psychotherapy at baseline is an important predictor for treatment outcome in patients treated with psychotherapy [26].

Regarding sertraline, matched patients had better outcomes than mismatched patients. However, more detailed analysis demonstrated only a statistical trend that the outcome of matched patients was superior to that of mismatched patients in week $10(\Delta=2.86$ points; $95 \% \mathrm{CI}$ : -0.22 to 5.93 points). In line with this finding, response and remission rates in matched and mismatched patients treated with sertraline did not significantly differ. Thus, there exists a certain additional benefit of getting the preferred treatment in the case of depressed primary care outpatients receiving sertraline ( $\triangle$ HAMD-17:2.9 points); however, this effect is smaller than the corresponding effect in patients treated with CBT-G.

Restrictively, it must be taken into account that initial preferences can change rapidly depending on initial experiences with the therapeutic intervention [27]. Since the FMP scale was only administered at the beginning of the study, we can only speculate about possible changes in attitudes over time. However, the unexpected finding that $40 \%$ of patients with the possibility of therapy choice who decided to get sertraline initially did not tend to prefer pharmacotherapy in the FMP-TP item might indicate changes in initial reservations concerning antidepressants, due to later extensive education about sertraline and its desired effects. Thus, the differences between matched and mismatched patients in response to sertraline might have been more pronounced if treatment preferences had been assessed after this information had been given.

The secondary research questions will be discussed below.

The analysis of the treatment preferences of 145 primary care patients suffering from mild-to-moderate depressive disorders revealed that most (58.6\%) favor a psychological treatment over a drug treatment, and 19.3\% were undetermined.

Indecisiveness and a negative treatment expectation could be symptoms of the depressive syndrome. The somewhat lower preference for drugs probably reflects the widespread prejudices about antidepressants [e.g. 28], especially in the general population [e.g. 2]. Surveys in 2 German cities revealed that about $80 \%$ of the population believe that antidepressants are addictive and about twothirds that they change one's personality [2].

In the present study, depressed primary care outpatients with a stronger preference for drugs did not significantly differ from those with a stronger preference for psychotherapy in demographic and clinical variables. Thus, it is unlikely that preference of drugs versus psychotherapy constitutes distinct clinical subgroups of patients suffering from depressive disorders.

The study design allowed us to address the question of whether patients getting their preferred treatment by choice (PC arm) have significant benefit in terms of treatment outcome, as compared to patients who receive their preferred treatment by chance, after randomization. This possibility can clearly be rejected. Thus, the benefits resulting from the act of choosing seem to be too small to result in considerable gains in terms of therapy outcome (as hypothesized by Leykin et al. [8]). More crucial seems to be fitting of the initial therapy preference and the received therapy.

The pronounced association between treatment preference and outcome as identified in the active treatment arms was less clear in the placebo arm. Insufficient statistical power might be the explanation for the lack of significant association between treatment preference and the intensity of therapeutic improvement in this group.

Receiving non-preferred therapy did not increase the probability of dropping out in our study since matched and mismatched patients did not significantly differ in the number of dropouts and the number of attended visits. So, the compliance to the received therapy does not seem to be influenced by the match or mismatch with initial treatment preference. Similar results were obtained by Leykin et al. [8].

\section{Limitations}

A general limitation of this study is the fact that only subjects who agreed to be randomized were included and that only patients who were randomized in the PC arm had the option to choose. Therefore, patients with preferences for a certain treatment strong enough to prompt them to refuse participation could not be investigated.

Another issue is related to the fact that CBT-G was offered as group psychotherapy in the context of the MinD study. In view of the fact that patients differ substantially in their attitudes toward individual compared to group 
psychotherapy, participants might have responded positively to the FMP-TP item in our study in general, yet had different attitudes toward group psychotherapy. This probably was the case as $40 \%$ of the patients in the preference arm who decided to receive sertraline had an initial preference for psychological treatment in general (but possibly not for group CBT-G resulting in a decision in favor of sertraline). However, this assumption is clearly speculative and can only be confirmed in studies using an item specifically addressing preference for group psychotherapy.

Further, we did not measure participants' pretreatment concerns about continuing antidepressant treatment in the presence of side effects. This can signal challenges to the completion of an acute phase treatment, as a recent study of Warden et al. [29] has shown. The use of the HAMD-17 as a measure of outcome might be another limitation [30]. Although the HAMD-17 is the most commonly used rating scale for depression severity [30], the ability of the HAMD-17 in judging depression severity is still doubtful [31].

The median HAMD-17 score of the patients enrolled was markedly below most clinical trials in major depressive disorder. Therefore, significant differences between active treatment and placebo treatment are difficult to find. However, minor depression is common in primary care and is associated with high levels of functional impairment [32]. Furthermore, there are convincing arguments that the benefit of antidepressants in mild depression is considerably larger than that suggested by placebo/ active treatment differences in clinical trials [33]. Thus, it is important to consider this large group of high utilizers of primary care services [34].

\section{Conclusion}

The treatment preference of a depressed patient for psychotherapy or pharmacotherapy is a relevant factor influencing treatment outcome. The outcome difference between receiving pharmaco- or psychotherapy in line with or contrary to the individual treatment preference is clearly of clinical significance.

Therefore, treatment preferences have to be incorporated as an important element in clinical decisions about treatment strategies. In this context, integration of patients' treatment preferences in shared decision making interventions in primary care of depression [35] is of special interest. Future studies should account for mediating factors like treatment satisfaction and provide the possibility to assess possible changes of treatment preferences in the course of therapy.

\section{Acknowledgments}

This project was supported by the German Ministry for Education and Research in Bonn within the promotional emphasis 'German Research Network on Depression and Suicidality'. The funding agency had no role in the design and conduct of the study; collection, management, analysis, or interpretation of the data; or preparation, review, or approval of the article.

\section{Conflict of Interest}

The authors report no financial or other relations relevant to the subject of this article.

\section{References}

1 Dwight-Johnson M, Sherbourne CD, Liao D, Wells KB: Treatment preferences among depressed primary care patients. J Gen Intern Med 2000;15:527-534.

2 Hegerl U, Althaus D, Stefanek J: Public attitudes towards treatment of depression: Effects of an information campaign. Pharmacopsychiatry 2003;36:288-291.

3 Jorm AF, Christensen H, Medway J, Korten AE, Jacomb PA, Rodgers B: Public belief systems about the helpfulness of interventions for depression: Associations with history of depression and professional help-seeking. Soc Psychiatry Psychiatr Epidemiol 2000;35: 211-219.
4 Thornett A: Assessing the effect of patient and prescriber preference in trials of treatment of depression in general practice. Med Sci Monit 2001;7:1086-1091.

5 Bedi N, Chilvers C, Churchill R, Dewey M, Duggan C, Fielding K, Gretton V, Miller P, Harrison G, Lee A, Williams I: Assessing effectiveness of treatment of depression in primary care. Partially randomised preference trial. Br J Psychiatry 2000;177:312-318.

6 Ward E, King M, Lloyd M, Bower P, Sibbald B, Farrelly S, Gabbay M, Tarrier N, Addington-Hall J: Randomised controlled trial of non-directive counselling, cognitive-behaviour therapy, and usual general practitioner care for patients with depression. I: Clinical effectiveness. BMJ 2000;321:1383-1388.
7 King M, Nazareth I, Lampe F, Bower P, Chandler M, Morou M, Sibbald B, Lai R: Impact of participant and physician intervention preferences on randomized trials: A systematic review. Jama 2005;293:1089-1099.

8 Leykin Y, DeRubeis RJ, Gallop R, Amsterdam JD, Shelton RC, Hollon SD: The relation of patients' treatment preferences to outcome in a randomized clinical trial. Behav Ther 2007;38:209-217.

9 DeRubeis RJ, Hollon SD, Amsterdam JD, Shelton RC, Young PR, Salomon RM, O'Reardon JP, Lovett ML, Gladis MM, Brown LL, Gallop R: Cognitive therapy vs medications in the treatment of moderate to severe depression. Arch Gen Psychiatry 2005;62:409-416. 
10 Kocsis JH, Leon AC, Markowitz JC, Manber R, Arnow B, Klein DN, Thase ME: Patient preference as a moderator of outcome for chronic forms of major depressive disorder treated with nefazodone, cognitive behavioral analysis system of psychotherapy, or their combination. J Clin Psychiatry 2009; 70:354-361.

11 Pyne JM, Rost KM, Farahati F, Tripathi SP, Smith J, Williams DK, Fortney J, Coyne JC: One size fits some: The impact of patient treatment attitudes on the cost-effectiveness of a depression primary-care intervention. Psychol Med 2005;35:839-854.

12 Raue PJ, Schulberg HC, Heo M, Klimstra S, Bruce ML: Patients' depression treatment preferences and initiation, adherence, and outcome: A randomized primary care study. Psychiatr Serv 2009;60:337-343.

13 Hegerl U, Hautzinger M, Mergl R, Kohnen R, Schütze M, Scheunemann W, Allgaier AK, Coyne J, Henkel V: Effects of pharmacotherapy and psychotherapy in depressed primary-care patients: A randomized, controlled trial including a patients' choice arm. Int J Neuropsychopharmacol 2010;13:31-44.

14 Hautzinger M: Kognitive Verhaltenstherapie bei Depressionen [cognitive behaviour therapy of depression], ed 6th. Weinheim, Beltz/ PVU, 2003.

15 Hamilton M: A rating scale for depression. J Neurol Neurosurg Psychiatry 1960;23:5662.

16 American Psychiatric Association: Diagnostic and statistical manual of mental disorders (dsm-iv), ed 4th. Washington, DC, American Psychiatric Association, 1994.

17 World Medical Association: World medical association declaration of helsinki. Recommendations guiding physicians in biomedical research involving human subjects. Jama 1997;277:925-926.
18 Wittchen $\mathrm{H}-\mathrm{U}$, Pfister $\mathrm{H}$ : Instruktionsmanual zur Durchführung von DIA-X Interviews [manual for conducting DIA-X interviews]. Frankfurt am Main, Swets Test Services, 1997.

19 World Health Organization: Composite international diagnostic interview, version 1.1. Geneva, World Health Organisation, 1993.

20 Schneider W, Basler H-D, Beisenherz B: FMP. Fragebogen zur Messung der Psychotherapiemotivation [fmp. Inventory to measure psychotherapy motivation]. Weinheim, Beltz, 1989.

21 Twisk J: Applied longitudinal data analysis for epidemiology: A practical guide. New York, Cambridge University Press, 2003.

22 Gueorguieva R, Krystal JH: Move over anova: Progress in analyzing repeated-measures data and its reflection in papers published in the archives of general psychiatry. Arch Gen Psychiatry 2004;61:310-317.

23 Erdfelder E, Faul F, Buchner A: Gpower: A general power analysis program. Behavior Research Methods, Instruments, \& Computers 1996;28:1-11.

24 SPSS Inc: SPSS software, version 12.0. Chicago, Illinois, SPSS Inc., 2008.

25 Stata: Stata statistical software. Release 8.0. College Station, Texas, Stata Corporation, 2003.

26 Schneider W, Klauer T, Janssen PL, Tetzlaff M: Zum Einfluss der Psychotherapiemotivation auf den Psychotherapieverlauf [influence of psychotherapy motivation on the course of psychotherapy]. Nervenarzt 1999; 70:240-249.

27 Bower P, King M, Nazareth I, Lampe F, Sibbald B: Patient preferences in randomised controlled trials: Conceptual framework and implications for research. Soc Sci Med 2005; 61:685-695.

28 Allgaier AK, Kramer D, Mergl R, Hegerl $\mathrm{U}$ : Wissens- und Einstellungsänderung zu Depression und Suizidalitat bei Altenpflegekräften: Evaluation eines Fortbildungsprojektes. [improvement of knowledge and attitudes towards depression and suicidality in geriatric caregivers: evaluation of an advanced training program]. Z Gerontol Geriatr 2009;42:228-235.
29 Warden D, Trivedi MH, Wisniewski SR, Lesser IM, Mitchell J, Balasubramani GK, Fava M, Shores-Wilson K, Stegman D, Rush AJ: Identifying risk for attrition during treatment for depression. Psychother Psychosom 2009;78:372-379.

30 Bech P: Fifty years with the hamilton scales for anxiety and depression. A tribute to Max Hamilton. Psychother Psychosom 2009;78: 202-211.

31 Maier W, Philipp M, Gerken A: [Dimensions of the hamilton depression scale. Factor analysis studies]. Eur Arch Psychiatry Neurol Sci 1985;234:417-422.

32 Howland RH, Schettler PJ, Rapaport MH, Mischoulon D, Schneider T, Fasiczka A, Delrahiem K, Maddux R, Lightfoot M, Nierenberg AA: Clinical features and functioning of patients with minor depression. Psychother Psychosom 2008;77:384-389.

33 Hegerl U, Mergl R: The clinical significance of antidepressant treatment effects cannot be derived from placebo-verum response differences. J Psychopharmacol 2010;24:445448 .

34 Ferrari S, Galeazzi GM, MacKinnon A, Rigatelli M: Frequent attenders in primary care: Impact of medical, psychiatric and psychosomatic diagnoses. Psychother Psychosom 2008;77:306-314

35 Loh A, Simon D, Wills CE, Kriston L, Niebling W, Harter M: The effects of a shared decision-making intervention in primary care of depression: A cluster-randomized controlled trial. Patient Educ Couns 2007;67: 324-332. 